\title{
Two-dimensional Laplace-transformed power series solution for solute transport in a radially convergent flow field
}

\author{
Jui-Sheng Chen ${ }^{\text {a }}$, Chen-Wuing Liu ${ }^{\text {b,*, }}$, Chung-Min Liao ${ }^{b}$ \\ ${ }^{a}$ Department of Environmental Engineering and Sanitation, Foo-Yin University, Kaohsiung 831, Taiwan, ROC \\ ${ }^{\mathrm{b}}$ Department of Bioenvironmental Systems Engineering, National Taiwan University, Taipei 10617, Taiwan, ROC
}

Received 16 July 2002; received in revised form 3 April 2003; accepted 21 May 2003

\begin{abstract}
This paper presents an analytical solution for two-dimensional non-axisymmetric solute transport in a radially convergent flow field. We applied a Laplace-transformed power series (LTPS) technique to solve the two-dimensional advection-dispersion equation in cylindrical coordinates. The solution is compared with a numerical solution to evaluate its robustness and accuracy. The applicable Péclet number range of the developed power series solution is also examined. Results show that the LTPS technique can effectively and accurately handle the two-dimensional radial advection-dispersion equation for a Péclet number up to 60 . The twodimensional power series solution is appropriate for hydrogeologic circumstances where temporally and spatially continuous solutions are demanded.
\end{abstract}

(C) 2003 Elsevier Ltd. All rights reserved.

Keywords: Radially convergent flow field; Laplace-transformed power series; Solute transport

\section{Introduction}

Tracer tests attempt to determine solute transport parameters, such as aquifer porosity, dispersion tensor and hydrogeological properties. A radially convergent tracer test facilitates the recovery of the injected mass, reduces the effect of apparent dispersion due to the flow field, and minimizes the influence of the natural hydraulic gradient. Thus, radially convergent tracer tests are particularly useful where transport, rather than hydraulic properties, is desired. Predictive and interpretive models are available for radially convergent tracer tests $[1,2,12-15,19,20]$. These studies are commonly limited to the analysis of breakthrough curves in the extraction well, although new sampling technologies are available for concentration measurements at arbitrary points in the field [11]. Because of the model complexity, the analysis is often reduced to an adjusted one-dimensional solution [17] instead of a more accurate two-dimensional transport model [22].

\footnotetext{
${ }^{*}$ Corresponding author. Tel.: +886-2-2362-6480; fax: +886-2-23639557.

E-mail address: 1cw@gwater.agec.ntu.edu.tw (C.-W. Liu).
}

The one-dimensional model generally considers longitudinal dispersion only. Solutions that consider transverse dispersion of points between extraction and injection wells are not available for tracer test analysis. Unlike a divergent flow tracer test, the convergent flow one does not have axial symmetry and the transverse dispersion could be important. The magnitude of the transverse dispersion coefficient influences both the region to which the pollution is extended, and the intensity of the pollution. In view of the importance of determining transverse dispersion, Chen et al. [3] presented a two-dimensional mathematical model in cylindrical coordinates that was solved via Laplace transform finitedifference method to illustrate non-axisymmetric tracer transport in a radially convergent flow field. In addition, a curve-fitting method involving a theoretical breakthrough curve at an intermediate point was proposed to evaluate transverse dispersivity, a quantity could not be determined from a one-dimensional model. The numerical solution developed by Chen et al. [3] has the propensity to be computationally cumbersome and expensive and, in the absence of interpolation, can be used to yield breakthrough curves only at discrete nodal points. An analytical solution can inherently provide a spatially continuous concentration distribution, thus is 
extremely useful in generating theoretical breakthrough curves at any observation point. Beyond its obvious importance in the studying solute transport for a tracer test in a radially convergent flow field, the radially convergent dispersion problem is distinguished by its being probably the simplest case for which the dispersion coefficient is a function of spatially varying velocity field. Accordingly, the two-dimensional analytical solution to the radially convergent dispersion problem can be a valuable means of evaluating the accuracy of computer codes that simulate two-dimensional solute transport in porous medium.

It is difficult to derive the analytical solution of the two-dimensional advection-dispersion equation in cylindrical coordinates due to the dependence of both the longitudinal and transverse dispersion coefficients on the spatially varying velocities. To our knowledge, the twodimensional analytical solution to the radial advectiondispersion equation in the real or Laplace domains is not currently available.

In this paper the problem is approached analytically to yield temporally and spatially continuous solutions. We used a Laplace-transformed power series (LTPS) technique [4] to solve the two-dimensional radial advection-dispersion equation in cylindrical coordinates. The analytical power series solution will be compared to the numerical solution of Chen et al. [3] to examine its robustness and accuracy and to determine the applicable ranges of the Péclet number. Moreover, the mathematical characteristics of the solutions are analyzed to illustrate the mathematical and convergence behavior of the power series functions.

\section{Problem formulation}

In this paper the problem of two-dimensional tracer transport in a radially convergent flow field is considered. Fig. 1 presents the conceptual configuration. For
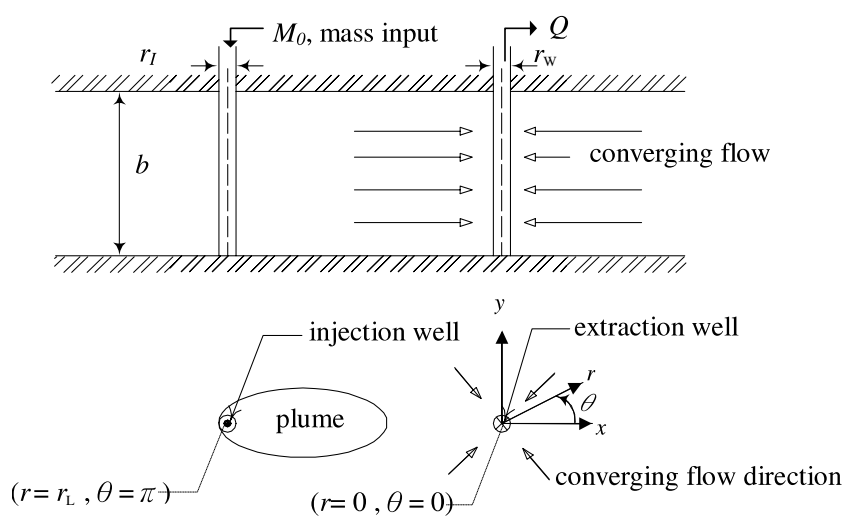

Fig. 1. Schematic diagram of a convergent tracer test. the sake of simplicity, the following assumptions are made:

1. A vertically oriented extraction well of finite diameter is located along the vertical axis and fully penetrates a homogeneous and isotropic aquifer of constant thickness.

2. A steady state, horizontal flow field, radially convergent and axially symmetric with respect to extraction well, is established prior to the start of tracer injection.

3. The tracer injection has no influence on the flow field.

Based on above assumptions the seepage velocity $V$ caused by extraction is described by

$V=-\frac{A}{r}$

where $A=Q / 2 \pi b \phi$ and $Q$ is volumetric rate of water withdrawal from the extraction well. The symbols $b$ and $\phi$ represent the aquifer thickness and effective porosity, respectively, while $r$ is the radial distance from the extraction well.

The two-dimensional advection-dispersion equation in a cylindrical coordinates is [22]

$\frac{a_{\mathrm{L}} A}{r} \frac{\partial^{2} C}{\partial^{2} r}+\frac{A}{r} \frac{\partial C}{\partial r}+\frac{a_{\mathrm{T}} A}{r^{3}} \frac{\partial^{2} C}{\partial^{2} \theta}=R \frac{\partial C}{\partial t}$

where $a_{\mathrm{L}}$ and $a_{\mathrm{T}}$ denote the longitudinal and transverse dispersivities, respectively, $R$ is a retardation factor, $t$ is time and $C$ is the solute concentration.

The aquifer's initial tracer concentration is assumed to be zero before starting the test:

$C(r, \theta, 0)=0$

The outlet boundary condition describes the solute transport between the extraction well and aquifer. The condition which takes into account the finite mixing volume effect in the well bore is

$\pi r_{\mathrm{W}}^{2} h_{\mathrm{W}} \frac{\partial C_{\mathrm{W}}(t)}{\partial t}=2 \pi r_{\mathrm{W}} \phi b a_{\mathrm{L}}\left|\frac{A}{r_{\mathrm{W}}}\right| \frac{\partial C(r, \theta, t)}{\partial r} \quad$ at $r=r_{\mathrm{W}}$

where $r_{\mathrm{W}}$ is the radius of the extraction well, $h_{\mathrm{W}}$ is its mixing length and $C_{\mathrm{W}}(t)$ denotes the tracer concentration in the well $[9,12]$.

The initial condition for (4) states that the well bore contains no contaminant before pumping:

$C_{\mathrm{W}}(t=0)=0$.

Additionally, it is reasonable to assume here that $C_{\mathrm{W}}(t)=C\left(r_{\mathrm{W}}, \theta, t\right)$.

Zlotnik and Logan [22] considered flow and transport in a ring-shaped domain centered at the extraction well and bounded by circles of radii $r=r_{\mathrm{I}}$ and $r^{*}=$ $r_{\mathrm{L}}-l\left(l \ll r_{\mathrm{L}}\right)$ in deriving the boundary condition at 
the injection well. The physical assumption is that advective transport dominates dispersive transport at a small distance $l \approx 5 r_{\text {I }}$ downstream in the discharge zone of the injection well. Therefore, the boundary condition including solute transport and the well bore effect at the injection well was formulated as [22]

$$
\begin{aligned}
a_{\mathrm{L}} & \frac{A}{r} \frac{\partial C(r, \theta, t)}{\partial r}+\frac{A}{r} C(r, \theta, t) \\
\quad & =\left\{\begin{array}{ll}
\frac{A}{r} C_{\mathrm{I}}(t) & \pi-\delta<\theta<\pi \\
0 & 0<\theta<\pi-\delta
\end{array} \quad r=r^{*} \approx r_{\mathrm{L}}\right.
\end{aligned}
$$

where $C_{\mathrm{I}}(t)$ is the concentration generated in the injection well and transported downstream through the narrow and short (a few well diameters) discharge zone by advection. This small zone with advection-dominated flow has an aperture angle of $2 \delta$. The aperture angle of this narrow zone at this distance $r^{*} \approx r_{\mathrm{L}}-l$ from the center of extraction well is

$2 \delta=\frac{2 \alpha r_{\mathrm{I}}}{r_{\mathrm{L}}}$

where $\alpha$ is a factor that defines the distortion of distances between the two most separated stream lines that enter (or leave) the injection well. This parameter can also depend on the skin effect for an injection well (Zlotnik and Logan [22]; Eq. (3)). For a uniform isotropic aquifer with a well without a skin, $\alpha=2$. For skins with high conductivity, $2 \leqslant \alpha \leqslant 4$, whereas for skins with low conductivity, $0<\alpha \leqslant 2$ [7].

It remains to determine the effluent concentration from the injection well in an ambient horizontal flow. Effluent concentration from the well with initial dissolved tracer mass $M_{0}$ satisfies a mass balance equation for the tracer in the borehole, namely

$-2 \alpha r_{\mathrm{I}} \phi b\left|V\left(r_{\mathrm{L}}\right)\right| C_{\mathrm{I}}=\pi r_{\mathrm{I}}^{2} h_{\mathrm{I}} \frac{\mathrm{d} C_{\mathrm{I}}}{\mathrm{d} t}$

$C_{\mathrm{I}}(0)=\frac{M_{0}}{\pi r_{\mathrm{I}}^{2} h_{\mathrm{I}}}=C_{0}$

where $h_{\mathrm{I}}$ is the mixing length of injection well (Zlotnik and Logan [22]; Eq. (6)).

After integration, the known effluent concentration $C_{\mathrm{I}}(t)$ can be substituted into boundary condition (6). The physics of the problem stipulates that $C$ is a singlevalued function in $r$ and $\theta$ coordinates. In addition, $C$ is a continuous and symmetrical function across $\theta=0$ and $\theta=\pi$. Thus, boundary conditions in the transverse directions are as follows:

$\frac{\partial C(r, 0, t)}{\partial \theta}=0$

$$
\frac{\partial C(r, \pi, t)}{\partial \theta}=0
$$

Table 1

Dimensionless parameters used in this study

\begin{tabular}{ll}
\hline Dimensionless quantity & Expression \\
\hline Time & $t_{\mathrm{D}}=\frac{Q t}{\pi h \phi\left(r_{\mathrm{L}}^{2}-r_{\mathrm{W}}^{2}\right)}$ \\
Distance & $r_{\mathrm{D}}=\frac{r}{r_{\mathrm{L}}}$ \\
Extraction well radius & $r_{\mathrm{WD}}=\frac{r_{\mathrm{W}}}{r_{\mathrm{L}}}$ \\
Injection well radius & $r_{\mathrm{ID}}=\frac{r_{\mathrm{I}}}{r_{\mathrm{L}}}$ \\
Péclet number & $P e=\frac{r_{\mathrm{L}}}{a_{\mathrm{L}}}$ \\
Transverse dispersivity & $X=\frac{a_{\mathrm{T}}}{a_{\mathrm{L}}}$ \\
Extraction well mixing factor & $\mu_{\mathrm{W}}=\frac{r_{\mathrm{W}}^{2} h_{\mathrm{W}}}{\phi h\left(r_{\mathrm{L}}^{2}-r_{\mathrm{W}}^{2}\right)}$ \\
Injection well mixing factor & $\mu_{\mathrm{I}}=\frac{r_{\mathrm{I}} r_{\mathrm{L}} h_{\mathrm{I}}}{\phi h\left(r_{\mathrm{L}}^{2}-r_{\mathrm{W}}^{2}\right)}$ \\
\hline
\end{tabular}

Dimensionless variables are defined in a manner similar to that used by Chen et al. [3]. Following Moench [12] and substituting the definition given in Table 1 into Eq. (2), the dimensionless transport equation is presented in the following form:

$\frac{1}{P e} \frac{1}{r_{\mathrm{D}}} \frac{\partial^{2} C}{\partial r_{\mathrm{D}}^{2}}+\frac{1}{r_{\mathrm{D}}} \frac{\partial C}{\partial r_{\mathrm{D}}}+\frac{1}{P e} \frac{a_{\mathrm{D}}}{r_{\mathrm{D}}^{3}} \frac{\partial^{2} C}{\partial \theta^{2}}=\frac{2 R}{1-r_{\mathrm{WD}}^{2}} \frac{\partial C}{\partial t_{\mathrm{D}}}$

Consequently, the initial and boundary conditions (3)(11) become

$C\left(r_{\mathrm{D}}, \theta, 0\right)=0$

$\mu_{\mathrm{W}} \frac{\partial C_{\mathrm{W}}\left(t_{\mathrm{D}}\right)}{\partial t_{\mathrm{D}}}=\frac{1}{P e} \frac{\partial C\left(r_{\mathrm{D}}, \theta, t_{\mathrm{D}}\right)}{\partial r_{\mathrm{D}}} \quad$ at $r=r_{\mathrm{W}}$

$C_{\mathrm{W}}(0)=0$

$\frac{1}{P e} \frac{\partial C\left(r_{\mathrm{D}}, \theta, t_{\mathrm{D}}\right)}{\partial r_{\mathrm{D}}}+C\left(r_{\mathrm{D}}, \theta, t_{\mathrm{D}}\right)$

$= \begin{cases}C_{\mathrm{I}}\left(t_{\mathrm{D}}\right) & \pi-\delta<\theta<\pi \\ 0 & 0<\theta<\pi-\delta\end{cases}$

$-C_{\mathrm{I}}=\mu_{\mathrm{I}} \frac{\mathrm{d} C_{\mathrm{I}}}{\mathrm{d} t_{\mathrm{D}}}$

$C_{\mathrm{I}}(0)=C_{0}$

$\frac{\partial C\left(r_{\mathrm{D}}, 0, t_{\mathrm{D}}\right)}{\partial \theta}=0$

$\frac{\partial C\left(r_{\mathrm{D}}, \pi, t_{\mathrm{D}}\right)}{\partial \theta}=0$

This study adopts the LTPS technique to solve the governing equation and boundary conditions (12)-(20). The LTPS has been successfully applied to solve the one-dimensional radial partial differential equation with variable-dependent coefficients [4]. The LTPS technique avoids the need to derive the full analytical solution. 
This technique is parsimonious and easy to code into a program. The detailed application of the LTPS technique is presented in Appendix A.

\section{Results and discussion}

\subsection{Verification of power series solution}

The obtained solution is compared with the numerical solution from the Laplace-transformed finite difference (LTFD) method [5] to demonstrate the accuracy of the LPTS solution. A hypothetical tracer test is defined for the purpose of the comparison. Table 2 provides a

Table 2

Descriptive simulation conditions and transport parameters of the hypothetical tracer test

\begin{tabular}{ll}
\hline Parameter & Test 1 \\
\hline Pumping rate $(Q), \mathrm{m}^{3} \mathrm{~min}^{-1}$ & 2 \\
Aquifer thickness $(h), \mathrm{m}$ & 10 \\
Effective porosity $(\phi)$, dimensionless & 0.2 \\
Radius of extraction well $\left(r_{\mathrm{W}}\right), \mathrm{m}$ & 0.1 \\
Extraction well mixing length $\left(h_{\mathrm{W}}\right), \mathrm{m}$ & 10 \\
Radius of injection well $\left(r_{\mathrm{I}}\right), \mathrm{m}$ & 0.1 \\
Injection well mixing length $\left(h_{\mathrm{I}}\right), \mathrm{m}$ & 10 \\
Distance to the injection well $\left(r_{\mathrm{L}}\right), \mathrm{m}$ & 25 \\
Injected mass $(M), \mathrm{Kg}$ & 40 \\
Longitudinal dispersivity $\left(a_{\mathrm{L}}\right), \mathrm{m}$ & $25,2.5,0.42$ \\
Péclet Number Pe, dimensionless & $1,10,60$ \\
Transverse dispersivity $\left(a_{\mathrm{T}}\right), \mathrm{m}$ & $5,0.5,0.084$ \\
Dimensionless ratio of dispersivity $(X)$, & 0.2 \\
dimensionless &
\end{tabular}

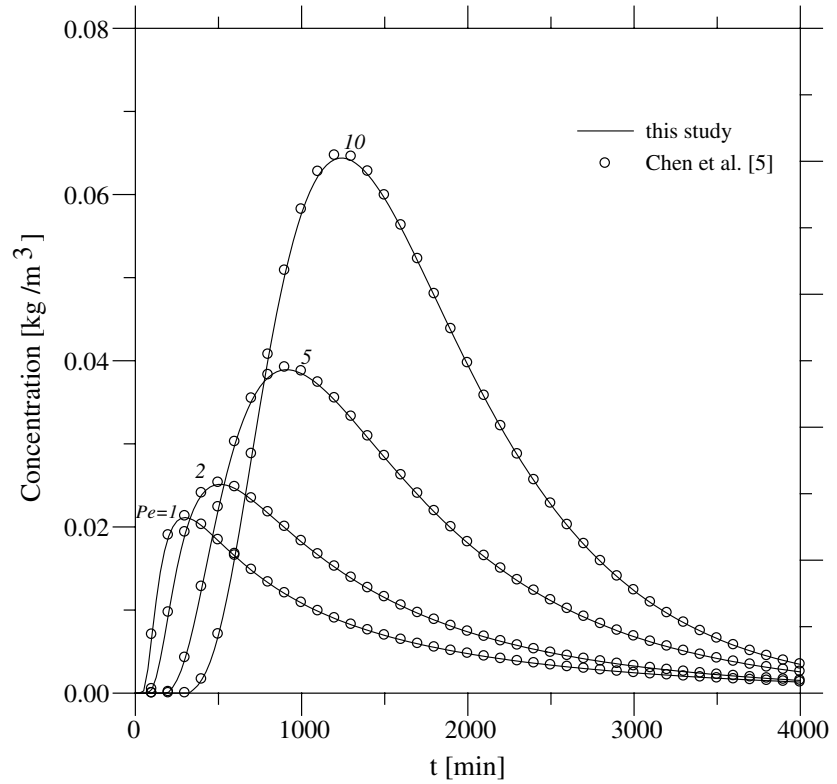

Fig. 2. Comparison of breakthrough curves at an observation well located at $r=5[\mathrm{~m}], \theta=\pi$ between the power series solution and numerical solution for Péclet numbers of $1,2,5$ and 10 in a radially convergent tracer test.

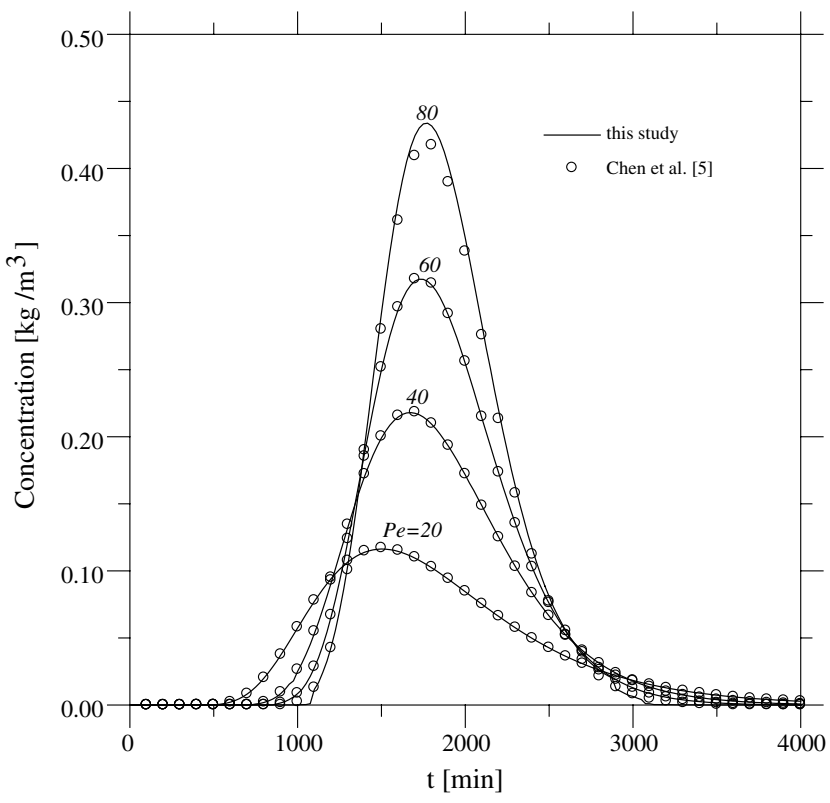

Fig. 3. Comparison of breakthrough curves at an observation well located at $r=5[\mathrm{~m}], \theta=\pi$ between the power series solution and numerical solution for Péclet numbers of 20,40,60 and 80 in a radially convergent tracer test.

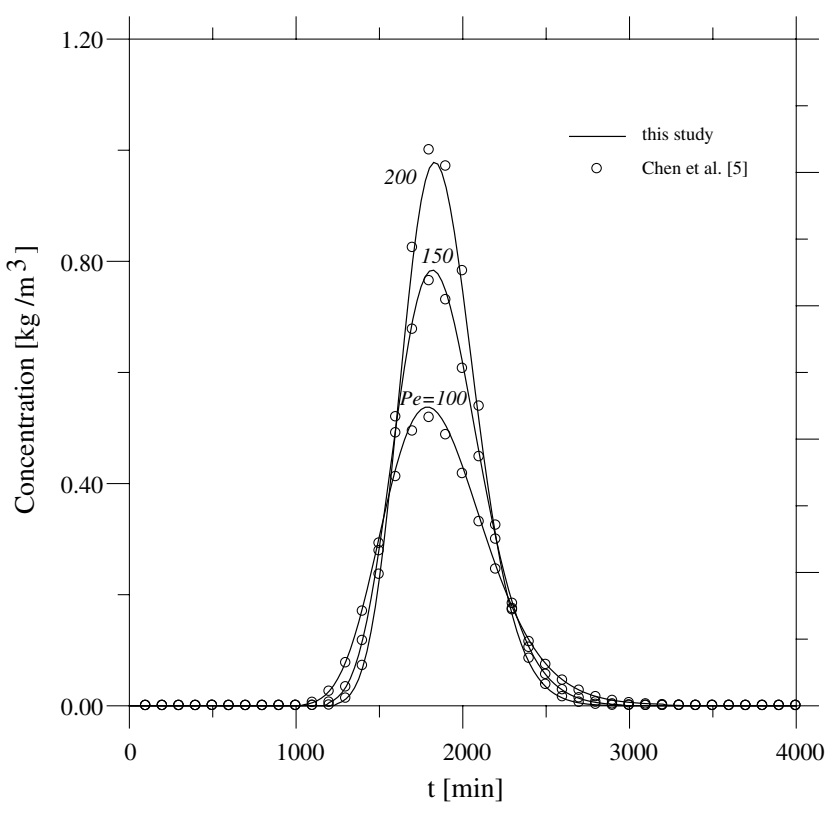

Fig. 4. Comparison of breakthrough curves at an observation well located at $r=5[\mathrm{~m}], \theta=\pi$ between the power series solution and numerical solution for Péclet numbers of 100,150 and 200 in a radially convergent tracer test.

summary of simulation conditions and transport parameters for the hypothetical tracer test. Figs. 2-4 plot breakthrough curves at an observation well located at $r=5 \mathrm{~m}, \theta=\pi$ using various Péclet numbers, and compares them to the numerical solution of Chen et al. 
[5]. The extraction well mixing and injection well mixing factors are set to zero so that well bore mixing exerts no influence on the breakthrough curves. Concentration breakthrough curves obtained from the power series solution agree well with those obtained from the numerical solution for Péclet numbers smaller than 60 . Additionally, the comparison reveals that the breakthrough curves obtained with LTPS are shaped roughly the same as those computed with LTFD for rising limbs and spreading tails for Péclet numbers greater than 60 , but a noticeable discrepancy occurs between the two solutions at the peak concentrations of the breakthrough curves for Péclet numbers greater than 60 .

The comparison of the two solutions reveals that the LPTS technique can effectively and accurately solve the two-dimensional, radial advection-dispersion equation. However, it is found that the power series method did not match the numerical solution for Péclet numbers greater than 60 . Such conditions correspond to an advective-dominated solute transport and yield breakthrough curves of steep fronts. Transport in a single fracture or in a particularly homogeneous granular aquifer may involve large Péclet numbers [18]. Efforts to determine solution correctness for Péclet numbers greater than 60 will be addressed in a future study.

\subsection{Mathematical behavior of power series functions}

The LPTS solution derived from the Appendix A includes two new functions. These two functions are in the form of infinite series, terms of which can be straightforwardly evaluated. We have to consider, however, the number of terms needed to produce accurate results. Chen et al. [4] illustrated that, for a fixed tolerance, the required numbers to be summed generally increase with the Péclet numbers.

We have performed the computation for various $r_{\mathrm{D}}$ and $n$ at fixed $s=3$ of the two new functions to examine the mathematical characteristics of the two new infinite series (some part of computational results are provided in the Appendix B). The values of the function $Z_{1}\left(r_{\mathrm{D}}, n, s\right)$ increase with increasing $r_{\mathrm{D}}$ and decrease with increasing $n$. The function values increase as Péclet number increases. The values of $\partial Z_{1}\left(r_{\mathrm{D}}, n, s\right) / \partial r_{\mathrm{D}}$ increase as $r_{\mathrm{D}}$ increases and decrease as $n$ increases. For a Péclet number of unity, however, the values of $\partial Z_{1}\left(r_{\mathrm{D}}, n, s\right) / \partial r_{\mathrm{D}}$ increase with $n$ at $r_{\mathrm{D}}=1$. The plot of $Z_{2}\left(r_{\mathrm{D}}, n, s\right)$ versus $r_{\mathrm{D}}$ behaves differently from that of $Z_{1}\left(r_{\mathrm{D}}, n, s\right): Z_{2}\left(r_{\mathrm{D}}, n, s\right)$ decreases with increasing $r_{\mathrm{D}}$ and behaves irregularly with respect to $n$. For example, $Z_{2}\left(r_{\mathrm{D}}, n, s\right)$ increases with $n$ at $r_{\mathrm{D}}=0.2$, however, no

Table 3

The values of $Z_{2}\left(r_{\mathrm{D}}, n, s\right)$ for various $r_{\mathrm{D}}$ and $n$ at $s=3(P e=1)$

\begin{tabular}{|c|c|c|c|c|c|}
\hline$n$ & $r_{\mathrm{D}}=0.2$ & $r_{\mathrm{D}}=0.4$ & $r_{\mathrm{D}}=0.6$ & $r_{\mathrm{D}}=0.8$ & $r_{\mathrm{D}}=1.0$ \\
\hline 0 & $2.011 \times 10^{12}$ & $4.156 \times 10^{12}$ & $6.765 \times 10^{12}$ & $1.045 \times 10^{12}$ & $1.633 \times 10^{12}$ \\
\hline 1 & $3.298 \times 10^{12}$ & $5.563 \times 10^{12}$ & $8.165 \times 10^{12}$ & $1.195 \times 10^{12}$ & $1.819 \times 10^{12}$ \\
\hline 2 & $8.155 \times 10^{12}$ & $9.896 \times 10^{12}$ & $1.286 \times 10^{12}$ & $1.837 \times 10^{12}$ & $2.852 \times 10^{12}$ \\
\hline 3 & $2.431 \times 10^{12}$ & $3.482 \times 10^{12}$ & $7.013 \times 10^{12}$ & $1.412 \times 10^{12}$ & $2.725 \times 10^{12}$ \\
\hline 4 & $5.608 \times 10^{12}$ & $2.439 \times 10^{12}$ & $1.162 \times 10^{12}$ & $1.383 \times 10^{12}$ & $-1.138 \times 10^{12}$ \\
\hline 5 & $1.537 \times 10^{12}$ & $4.535 \times 10^{12}$ & $1.964 \times 10^{12}$ & $6.649 \times 10^{12}$ & $-5.521 \times 10^{12}$ \\
\hline 6 & $4.218 \times 10^{12}$ & $8.213 \times 10^{12}$ & $3.065 \times 10^{12}$ & $1.597 \times 10^{12}$ & $1.308 \times 10^{12}$ \\
\hline 7 & $1.160 \times 10^{12}$ & $1.468 \times 10^{12}$ & $4.248 \times 10^{12}$ & $1.749 \times 10^{12}$ & $1.043 \times 10^{12}$ \\
\hline 8 & $3.193 \times 10^{12}$ & $2.623 \times 10^{12}$ & $5.892 \times 10^{12}$ & $1.895 \times 10^{12}$ & $5.321 \times 10^{12}$ \\
\hline 9 & $8.802 \times 10^{12}$ & $4.685 \times 10^{12}$ & $8.218 \times 10^{12}$ & $2.301 \times 10^{12}$ & $8.057 \times 10^{12}$ \\
\hline 10 & $2.428 \times 10^{12}$ & $8.363 \times 10^{12}$ & $1.141 \times 10^{12}$ & $2.689 \times 10^{12}$ & $8.318 \times 10^{12}$ \\
\hline 11 & $6.702 \times 10^{12}$ & $1.493 \times 10^{12}$ & $1.583 \times 10^{12}$ & $3.132 \times 10^{12}$ & $8.608 \times 10^{12}$ \\
\hline 12 & $1.850 \times 10^{12}$ & $2.664 \times 10^{12}$ & $2.193 \times 10^{12}$ & $3.636 \times 10^{12}$ & $8.733 \times 10^{12}$ \\
\hline 13 & $5.111 \times 10^{12}$ & $4.756 \times 10^{12}$ & $3.037 \times 10^{12}$ & $4.216 \times 10^{12}$ & $8.844 \times 10^{12}$ \\
\hline 14 & $1.412 \times 10^{12}$ & $8.488 \times 10^{12}$ & $4.204 \times 10^{12}$ & $4.882 \times 10^{12}$ & $8.940 \times 10^{12}$ \\
\hline 15 & $3.901 \times 10^{12}$ & $-1.006 \times 10^{12}$ & $-5.975 \times 10^{12}$ & $-1.092 \times 10^{12}$ & $-1.065 \times 10^{12}$ \\
\hline 16 & $1.079 \times 10^{12}$ & $2.704 \times 10^{12}$ & $8.045 \times 10^{12}$ & $6.529 \times 10^{12}$ & $9.091 \times 10^{12}$ \\
\hline 17 & $2.982 \times 10^{12}$ & $4.826 \times 10^{12}$ & $1.113 \times 10^{12}$ & $7.544 \times 10^{12}$ & $9.152 \times 10^{12}$ \\
\hline 18 & $8.243 \times 10^{12}$ & $8.613 \times 10^{12}$ & $1.539 \times 10^{12}$ & $8.713 \times 10^{12}$ & $9.205 \times 10^{12}$ \\
\hline 19 & $2.279 \times 10^{12}$ & $1.537 \times 10^{12}$ & $2.127 \times 10^{12}$ & $1.006 \times 10^{12}$ & $9.252 \times 10^{12}$ \\
\hline 20 & $6.302 \times 10^{12}$ & $2.744 \times 10^{12}$ & $2.941 \times 10^{12}$ & $1.161 \times 10^{12}$ & $9.294 \times 10^{12}$ \\
\hline 21 & $1.743 \times 10^{12}$ & $4.898 \times 10^{12}$ & $4.065 \times 10^{12}$ & $1.340 \times 10^{12}$ & $9.331 \times 10^{12}$ \\
\hline 22 & $4.820 \times 10^{12}$ & $8.742 \times 10^{12}$ & $5.618 \times 10^{12}$ & $1.546 \times 10^{12}$ & $9.365 \times 10^{12}$ \\
\hline 23 & $1.333 \times 10^{12}$ & $1.560 \times 10^{12}$ & $7.765 \times 10^{12}$ & $1.783 \times 10^{12}$ & $9.396 \times 10^{12}$ \\
\hline 24 & $3.687 \times 10^{12}$ & $2.785 \times 10^{12}$ & $1.073 \times 10^{12}$ & $2.056 \times 10^{12}$ & $9.423 \times 10^{12}$ \\
\hline 25 & $1.020 \times 10^{12}$ & $4.972 \times 10^{12}$ & $1.483 \times 10^{12}$ & $2.371 \times 10^{12}$ & $9.448 \times 10^{12}$ \\
\hline 26 & $2.821 \times 10^{12}$ & $8.874 \times 10^{12}$ & $2.050 \times 10^{12}$ & $2.733 \times 10^{12}$ & $9.472 \times 10^{12}$ \\
\hline 27 & $7.803 \times 10^{12}$ & $1.584 \times 10^{12}$ & $2.832 \times 10^{12}$ & $3.151 \times 10^{12}$ & $9.493 \times 10^{12}$ \\
\hline 28 & $2.159 \times 10^{12}$ & $2.828 \times 10^{12}$ & $3.914 \times 10^{12}$ & $3.632 \times 10^{12}$ & $9.513 \times 10^{12}$ \\
\hline 29 & $5.971 \times 10^{12}$ & $5.047 \times 10^{12}$ & $5.408 \times 10^{12}$ & $4.187 \times 10^{12}$ & $9.531 \times 10^{12}$ \\
\hline 30 & $1.652 \times 10^{12}$ & $9.009 \times 10^{12}$ & $7.472 \times 10^{12}$ & $4.826 \times 10^{12}$ & $9.548 \times 10^{12}$ \\
\hline
\end{tabular}


Table 4

The values of $Z_{2}\left(r_{\mathrm{D}}, n, s\right)$ for various $r_{\mathrm{D}}$ and $n$ at $s=3(P e=10)$

\begin{tabular}{|c|c|c|c|c|c|}
\hline$n$ & $r_{\mathrm{D}}=0.2$ & $r_{\mathrm{D}}=0.4$ & $r_{\mathrm{D}}=0.6$ & $r_{\mathrm{D}}=0.8$ & $r_{\mathrm{D}}=1.0$ \\
\hline 0 & $2.440 \times 10^{12}$ & $9.286 \times 10^{12}$ & $3.875 \times 10^{12}$ & $1.897 \times 10^{12}$ & $1.077 \times 10^{12}$ \\
\hline 1 & $4.296 \times 10^{12}$ & $1.542 \times 10^{12}$ & $6.503 \times 10^{12}$ & $3.219 \times 10^{12}$ & $1.840 \times 10^{12}$ \\
\hline 2 & $1.941 \times 10^{12}$ & $8.793 \times 10^{12}$ & $4.112 \times 10^{12}$ & $2.122 \times 10^{12}$ & $1.239 \times 10^{12}$ \\
\hline 3 & $4.855 \times 10^{12}$ & $2.732 \times 10^{12}$ & $1.468 \times 10^{12}$ & $8.077 \times 10^{12}$ & $4.876 \times 10^{12}$ \\
\hline 4 & $5.203 \times 10^{12}$ & $7.234 \times 10^{12}$ & $3.858 \times 10^{12}$ & $2.283 \times 10^{12}$ & $1.442 \times 10^{12}$ \\
\hline 5 & $1.405 \times 10^{12}$ & $1.103 \times 10^{12}$ & $6.495 \times 10^{12}$ & $4.234 \times 10^{12}$ & $2.827 \times 10^{12}$ \\
\hline 6 & $3.871 \times 10^{12}$ & $1.379 \times 10^{12}$ & $8.004 \times 10^{12}$ & $5.801 \times 10^{12}$ & $4.135 \times 10^{12}$ \\
\hline 7 & $1.079 \times 10^{12}$ & $2.285 \times 10^{12}$ & $1.463 \times 10^{12}$ & $1.198 \times 10^{12}$ & $9.194 \times 10^{12}$ \\
\hline 8 & $3.001 \times 10^{12}$ & $1.814 \times 10^{12}$ & $-1.902 \times 10^{12}$ & $-2.072 \times 10^{12}$ & $-1.729 \times 10^{12}$ \\
\hline 9 & $8.350 \times 10^{12}$ & $3.677 \times 10^{12}$ & $9.640 \times 10^{12}$ & $5.640 \times 10^{12}$ & $5.049 \times 10^{12}$ \\
\hline 10 & $2.319 \times 10^{12}$ & $6.750 \times 10^{12}$ & $1.534 \times 10^{12}$ & $1.093 \times 10^{12}$ & $1.079 \times 10^{12}$ \\
\hline 11 & $6.435 \times 10^{12}$ & $1.237 \times 10^{12}$ & $3.938 \times 10^{12}$ & $4.288 \times 10^{12}$ & $4.693 \times 10^{12}$ \\
\hline 12 & $1.784 \times 10^{12}$ & $2.232 \times 10^{12}$ & $1.105 \times 10^{12}$ & $-4.840 \times 10^{12}$ & $-6.070 \times 10^{12}$ \\
\hline 13 & $4.946 \times 10^{12}$ & $4.049 \times 10^{12}$ & $1.970 \times 10^{12}$ & $-6.879 \times 10^{12}$ & $-1.188 \times 10^{12}$ \\
\hline 14 & $1.370 \times 10^{12}$ & $7.324 \times 10^{12}$ & $2.880 \times 10^{12}$ & $2.646 \times 10^{12}$ & $4.413 \times 10^{12}$ \\
\hline 15 & $-8.670 \times 10^{12}$ & $-1.007 \times 10^{12}$ & $-7.456 \times 10^{12}$ & $-1.996 \times 10^{12}$ & $-3.356 \times 10^{12}$ \\
\hline 16 & $1.051 \times 10^{12}$ & $2.383 \times 10^{12}$ & $5.792 \times 10^{12}$ & $1.844 \times 10^{12}$ & $-3.015 \times 10^{12}$ \\
\hline 17 & $2.911 \times 10^{12}$ & $4.290 \times 10^{12}$ & $8.185 \times 10^{12}$ & $3.655 \times 10^{12}$ & $-1.010 \times 10^{12}$ \\
\hline 18 & $8.061 \times 10^{12}$ & $7.713 \times 10^{12}$ & $1.153 \times 10^{12}$ & $4.720 \times 10^{12}$ & $-5.154 \times 10^{12}$ \\
\hline 19 & $2.232 \times 10^{12}$ & $1.386 \times 10^{12}$ & $1.620 \times 10^{12}$ & $6.011 \times 10^{12}$ & $3.659 \times 10^{12}$ \\
\hline 20 & $6.179 \times 10^{12}$ & $2.488 \times 10^{12}$ & $2.273 \times 10^{12}$ & $6.976 \times 10^{12}$ & $2.411 \times 10^{12}$ \\
\hline 21 & $1.710 \times 10^{12}$ & $4.464 \times 10^{12}$ & $3.184 \times 10^{12}$ & $8.250 \times 10^{12}$ & $2.502 \times 10^{12}$ \\
\hline 22 & $4.735 \times 10^{12}$ & $8.005 \times 10^{12}$ & $4.454 \times 10^{12}$ & $9.733 \times 10^{12}$ & $2.652 \times 10^{12}$ \\
\hline 23 & $1.311 \times 10^{12}$ & $1.435 \times 10^{12}$ & $6.223 \times 10^{12}$ & $1.146 \times 10^{12}$ & $5.241 \times 10^{12}$ \\
\hline 24 & $3.628 \times 10^{12}$ & $2.571 \times 10^{12}$ & $8.686 \times 10^{12}$ & $1.347 \times 10^{12}$ & $4.681 \times 10^{12}$ \\
\hline 25 & $1.004 \times 10^{12}$ & $4.606 \times 10^{12}$ & $1.211 \times 10^{12}$ & $1.580 \times 10^{12}$ & $4.761 \times 10^{12}$ \\
\hline 26 & $2.780 \times 10^{12}$ & $8.248 \times 10^{12}$ & $1.688 \times 10^{12}$ & $1.851 \times 10^{12}$ & $4.878 \times 10^{12}$ \\
\hline 27 & $7.693 \times 10^{12}$ & $1.477 \times 10^{12}$ & $2.350 \times 10^{12}$ & $2.166 \times 10^{12}$ & $5.018 \times 10^{12}$ \\
\hline 28 & $2.129 \times 10^{12}$ & $2.643 \times 10^{12}$ & $3.271 \times 10^{12}$ & $2.531 \times 10^{12}$ & $5.136 \times 10^{12}$ \\
\hline 29 & $5.894 \times 10^{12}$ & $4.730 \times 10^{12}$ & $4.550 \times 10^{12}$ & $2.955 \times 10^{12}$ & $5.252 \times 10^{12}$ \\
\hline 30 & $1.631 \times 10^{12}$ & $8.463 \times 10^{12}$ & $6.325 \times 10^{12}$ & $3.447 \times 10^{12}$ & $5.363 \times 10^{12}$ \\
\hline
\end{tabular}

definite rule can be concluded for $Z_{2}\left(r_{\mathrm{D}}, n, s\right)$ versus $n$ at other values of $r_{\mathrm{D}}$. Notably, an abnormally large value appears at $n=15$ for $Z_{2}\left(r_{\mathrm{D}}, n, s\right)$ and its first derivative, $\partial Z_{2}\left(r_{\mathrm{D}}, n, s\right) / \partial r_{\mathrm{D}}$. We have scrutinized thoroughly the computer program and confirm that this extraordinary value dose not result from any computation errors but from the inherent mathematical characteristic of the new power series function. Understanding the characteristic of abnormality involves a complicated mathematical analysis, which we do not pursue here. Tables 3-8 also show that the behavior of the developed two functions are fundamentally different from that of the special Airy function solution for the one-dimensional radial advection-dispersion equation.

\section{Conclusions}

This work presents a novel Laplace transform power series method to solve the two-dimensional variabledependent advection-dispersion differential equation in cylindrical coordinates, accounting for non-axisymmetrical transport during a convergent radial tracer test. The exact analytical solution in Laplace transform do- main is derived. The new solution is compared with the numerical solution to verify its accuracy. Results show that the two solutions produce the same results for Péclet numbers smaller than 60 . Notably, it is found that the two solutions yield a noticeable difference at an intermediate time for Péclet numbers greater than 60 . We suggest further mathematical analysis and verification of the new solution for Péclet numbers greater than 60 are needed. The novel two-dimensional power series solution is useful for quantitative hydrogeologic problems where temporally and spatially continuous solutions are demanded.

\section{Acknowledgements}

The authors would like to acknowledge the National Science Council of the Republic of China for financially supporting this work under Grant no. NSC 90-2313B-242-004. Dr. G. Cassiani and the other anonymous reviewer are also appreciated for their constructive suggestions to improve this paper. 
Table 5

The values of $Z_{2}\left(r_{\mathrm{D}}, n, s\right)$ for various $r_{\mathrm{D}}$ and $n$ at $s=3(P e=60)$

\begin{tabular}{|c|c|c|c|c|c|}
\hline$n$ & $r_{\mathrm{D}}=0.2$ & $r_{\mathrm{D}}=0.4$ & $r_{\mathrm{D}}=0.6$ & $r_{\mathrm{D}}=0.8$ & $r_{\mathrm{D}}=1.0$ \\
\hline 0 & $7.424 \times 10^{12}$ & $4.172 \times 10^{12}$ & $2.934 \times 10^{12}$ & $2.562 \times 10^{12}$ & $2.760 \times 10^{12}$ \\
\hline 1 & $2.148 \times 10^{12}$ & $1.229 \times 10^{12}$ & $8.689 \times 10^{12}$ & $7.608 \times 10^{12}$ & $8.208 \times 10^{12}$ \\
\hline 2 & $4.393 \times 10^{12}$ & $2.654 \times 10^{12}$ & $1.908 \times 10^{12}$ & $1.683 \times 10^{12}$ & $1.824 \times 10^{12}$ \\
\hline 3 & $-7.808 \times 10^{12}$ & $-5.157 \times 10^{12}$ & $-3.809 \times 10^{12}$ & $-3.405 \times 10^{12}$ & $-3.717 \times 10^{12}$ \\
\hline 4 & $1.395 \times 10^{12}$ & $1.040 \times 10^{12}$ & $7.976 \times 10^{12}$ & $7.259 \times 10^{12}$ & $8.007 \times 10^{12}$ \\
\hline 5 & $1.265 \times 10^{12}$ & $1.096 \times 10^{12}$ & $8.823 \times 10^{12}$ & $8.217 \times 10^{12}$ & $9.186 \times 10^{12}$ \\
\hline 6 & $-1.725 \times 10^{12}$ & $-1.786 \times 10^{12}$ & $-1.524 \times 10^{12}$ & $-1.460 \times 10^{12}$ & $-1.659 \times 10^{12}$ \\
\hline 7 & $-2.387 \times 10^{12}$ & $-3.023 \times 10^{12}$ & $-2.762 \times 10^{12}$ & $-2.734 \times 10^{12}$ & $-3.166 \times 10^{12}$ \\
\hline 8 & $9.386 \times 10^{12}$ & $1.485 \times 10^{12}$ & $1.466 \times 10^{12}$ & $1.508 \times 10^{12}$ & $1.785 \times 10^{12}$ \\
\hline 9 & $-6.929 \times 10^{12}$ & $-1.406 \times 10^{12}$ & $-1.514 \times 10^{12}$ & $-1.624 \times 10^{12}$ & $-1.971 \times 10^{12}$ \\
\hline 10 & $-1.634 \times 10^{12}$ & $-4.306 \times 10^{12}$ & $-5.102 \times 10^{12}$ & $-5.739 \times 10^{12}$ & $-7.161 \times 10^{12}$ \\
\hline 11 & $-3.327 \times 10^{12}$ & $-1.159 \times 10^{12}$ & $-1.523 \times 10^{12}$ & $-1.805 \times 10^{12}$ & $-2.321 \times 10^{12}$ \\
\hline 12 & $-1.131 \times 10^{12}$ & $-5.585 \times 10^{12}$ & $-8.209 \times 10^{12}$ & $-1.029 \times 10^{12}$ & $-1.368 \times 10^{12}$ \\
\hline 13 & $-7.329 \times 10^{12}$ & $-6.020 \times 10^{12}$ & $-9.969 \times 10^{12}$ & $-1.328 \times 10^{12}$ & $-1.830 \times 10^{12}$ \\
\hline 14 & $3.234 \times 10^{12}$ & $-2.984 \times 10^{12}$ & $-5.606 \times 10^{12}$ & $-7.966 \times 10^{12}$ & $-1.141 \times 10^{12}$ \\
\hline 15 & $-4.565 \times 10^{12}$ & $-5.393 \times 10^{12}$ & $-1.157 \times 10^{12}$ & $-1.762 \times 10^{12}$ & $-2.630 \times 10^{12}$ \\
\hline 16 & $5.215 \times 10^{12}$ & $-6.696 \times 10^{12}$ & $-1.652 \times 10^{12}$ & $-2.705 \times 10^{12}$ & $-4.218 \times 10^{12}$ \\
\hline 17 & $1.607 \times 10^{12}$ & $-1.621 \times 10^{12}$ & $-4.624 \times 10^{12}$ & $-8.176 \times 10^{12}$ & $-1.335 \times 10^{12}$ \\
\hline 18 & $4.624 \times 10^{12}$ & $2.896 \times 10^{12}$ & $9.607 \times 10^{12}$ & $1.841 \times 10^{12}$ & $3.156 \times 10^{12}$ \\
\hline 19 & $1.313 \times 10^{12}$ & $-1.323 \times 10^{12}$ & $-5.129 \times 10^{12}$ & $-1.069 \times 10^{12}$ & $-1.929 \times 10^{12}$ \\
\hline 20 & $3.739 \times 10^{12}$ & $-9.048 \times 10^{12}$ & $-4.123 \times 10^{12}$ & $-9.380 \times 10^{12}$ & $-1.785 \times 10^{12}$ \\
\hline 21 & $1.060 \times 10^{12}$ & $4.659 \times 10^{12}$ & $2.506 \times 10^{12}$ & $6.243 \times 10^{12}$ & $1.256 \times 10^{12}$ \\
\hline 22 & $2.999 \times 10^{12}$ & $1.296 \times 10^{12}$ & $8.262 \times 10^{12}$ & $2.261 \times 10^{12}$ & $4.821 \times 10^{12}$ \\
\hline 23 & $8.470 \times 10^{12}$ & $-6.716 \times 10^{12}$ & $-5.101 \times 10^{12}$ & $-1.539 \times 10^{12}$ & $-3.484 \times 10^{12}$ \\
\hline 24 & $2.388 \times 10^{12}$ & $5.900 \times 10^{12}$ & $5.296 \times 10^{12}$ & $1.766 \times 10^{12}$ & $4.255 \times 10^{12}$ \\
\hline 25 & $6.722 \times 10^{12}$ & $1.113 \times 10^{12}$ & $1.199 \times 10^{12}$ & $4.430 \times 10^{12}$ & $1.138 \times 10^{12}$ \\
\hline 26 & $1.890 \times 10^{12}$ & $1.467 \times 10^{12}$ & $1.893 \times 10^{12}$ & $7.775 \times 10^{12}$ & $2.135 \times 10^{12}$ \\
\hline 27 & $5.307 \times 10^{12}$ & $5.037 \times 10^{12}$ & $1.470 \times 10^{12}$ & $6.730 \times 10^{12}$ & $1.979 \times 10^{12}$ \\
\hline 28 & $1.489 \times 10^{12}$ & $1.808 \times 10^{12}$ & $2.005 \times 10^{12}$ & $1.025 \times 10^{12}$ & $3.235 \times 10^{12}$ \\
\hline 29 & $4.173 \times 10^{12}$ & $2.061 \times 10^{12}$ & $1.515 \times 10^{12}$ & $8.677 \times 10^{12}$ & $2.943 \times 10^{12}$ \\
\hline 30 & $1.168 \times 10^{12}$ & $3.187 \times 10^{12}$ & $1.651 \times 10^{12}$ & $1.062 \times 10^{12}$ & $3.878 \times 10^{12}$ \\
\hline
\end{tabular}

\section{Appendix A}

First, proceeding with the Laplace transform of Eq. (12) and its associated boundary conditions (13)-(20), with respect to $t_{\mathrm{D}}$, we obtain

$\frac{1}{P e} \frac{1}{r_{\mathrm{D}}} \frac{\partial^{2} G}{\partial r_{\mathrm{D}}^{2}}+\frac{1}{r_{\mathrm{D}}} \frac{\partial G}{\partial r_{\mathrm{D}}}+\frac{1}{P e} \frac{a_{\mathrm{D}}}{r_{\mathrm{D}}^{3}} \frac{\partial^{2} G}{\partial \theta^{2}}=\frac{2 R}{1-r_{\mathrm{WD}}^{2}} s G$

$\mu_{\mathrm{W}} s G=\frac{1}{P e} \frac{\partial G\left(r_{\mathrm{D}}, \theta, s\right)}{\partial r_{\mathrm{D}}} \quad$ at $r=r_{\mathrm{W}}$

$\frac{1}{P e} \frac{\partial G\left(r_{\mathrm{D}}, \theta, s\right)}{\partial r_{\mathrm{D}}}+G\left(r_{\mathrm{D}}, \theta, s\right)$

$$
=\left\{\begin{array}{ll}
G_{\mathrm{I}}(s) & \pi-\delta<\theta<\pi \\
0 & 0<\theta<\pi-\delta
\end{array} \text { at } r=r_{\mathrm{I}}\right.
$$

$-G_{\mathrm{I}}(s)=\mu_{\mathrm{I}}\left[s G_{\mathrm{I}}(s)-C_{0}\right]$

$\frac{\partial G\left(r_{\mathrm{D}}, 0, s\right)}{\partial \theta}=0$

$$
\frac{\partial G\left(r_{\mathrm{D}}, \pi, s\right)}{\partial \theta}=0
$$

where $s$ denotes the Laplace transform parameter and $G$ represents the Laplace transform of $C$, as defined by

$G\left(r_{\mathrm{D}}, \theta, s\right)=\int_{0}^{\infty} C\left(r_{\mathrm{D}}, \theta, t_{\mathrm{D}}\right) \mathrm{e}^{-s t_{\mathrm{D}}} \mathrm{d} t_{\mathrm{D}}$

$G_{\mathrm{I}}(s)=\int_{0}^{\infty} C_{\mathrm{I}}\left(t_{\mathrm{D}}\right) \mathrm{e}^{-s t_{\mathrm{D}}} \mathrm{d} t_{\mathrm{D}}$

The finite Fourier cosine transform with respect to $\theta$ of (A.1)-(A.6) gives

$$
\frac{1}{P e} \frac{1}{r_{\mathrm{D}}} \frac{\mathrm{d}^{2} W}{\mathrm{~d} r_{\mathrm{D}}^{2}}+\frac{1}{r_{\mathrm{D}}} \frac{\mathrm{d} W}{\mathrm{~d} r_{\mathrm{D}}}-\left(\frac{1}{P e} \frac{a_{\mathrm{D}} n^{2}}{r_{\mathrm{D}}^{3}}+\frac{2 R}{1-r_{\mathrm{WD}}^{2}} s\right) W=0
$$

$$
\mu_{\mathrm{W}} s W=\frac{1}{P e} \frac{\partial W\left(r_{\mathrm{D}}, n, s\right)}{\partial r_{\mathrm{D}}} \quad \text { at } r=r_{\mathrm{W}}
$$

$$
\frac{1}{P e} \frac{\partial W\left(r_{\mathrm{D}}, n, s\right)}{\partial r_{\mathrm{D}}}+W\left(r_{\mathrm{D}}, n, s\right)=F(n)
$$

where

$$
F(n)=\left\{\begin{array}{ll}
G_{\mathrm{I}}(s) \delta, & n=0 \\
G_{\mathrm{I}}(s)\left[\frac{(-1)^{n} \sin n \delta}{n}\right], & n=1,2,3 \ldots
\end{array} \quad G_{\mathrm{I}}(s)=\frac{\mu_{\mathrm{I}} C_{0}}{1+\mu_{\mathrm{I}} s}\right.
$$


Table 6

The values of $\partial Z_{2}\left(r_{\mathrm{D}}, n, s\right) / \partial r_{\mathrm{D}}$ for various $r_{\mathrm{D}}$ and $n$ at $s=3(P e=1)$

\begin{tabular}{|c|c|c|c|c|c|}
\hline$n$ & $r_{\mathrm{D}}=0.2$ & $r_{\mathrm{D}}=0.4$ & $r_{\mathrm{D}}=0.6$ & $r_{\mathrm{D}}=0.8$ & $r_{\mathrm{D}}=1.0$ \\
\hline 0 & $1.021 \times 10^{12}$ & $1.151 \times 10^{12}$ & $1.507 \times 10^{12}$ & $2.270 \times 10^{12}$ & $3.766 \times 10^{12}$ \\
\hline 1 & $-2.070 \times 10^{12}$ & $1.485 \times 10^{12}$ & $1.706 \times 10^{12}$ & $3.756 \times 10^{12}$ & $7.102 \times 10^{12}$ \\
\hline 2 & $-1.487 \times 10^{12}$ & $7.485 \times 10^{12}$ & $7.594 \times 10^{12}$ & $1.538 \times 10^{12}$ & $2.790 \times 10^{12}$ \\
\hline 3 & $-7.842 \times 10^{12}$ & $-1.633 \times 10^{12}$ & $-8.785 \times 10^{12}$ & $-8.487 \times 10^{12}$ & $-1.184 \times 10^{12}$ \\
\hline 4 & $-2.959 \times 10^{12}$ & $-3.581 \times 10^{12}$ & $-1.120 \times 10^{12}$ & $-6.144 \times 10^{12}$ & $-5.904 \times 10^{12}$ \\
\hline 5 & $-1.045 \times 10^{12}$ & $-7.988 \times 10^{12}$ & $-1.636 \times 10^{12}$ & $-2.491 \times 10^{12}$ & $5.105 \times 10^{12}$ \\
\hline 6 & $-3.521 \times 10^{12}$ & $-1.746 \times 10^{12}$ & $-2.954 \times 10^{12}$ & $-7.569 \times 10^{12}$ & $-1.180 \times 10^{12}$ \\
\hline 7 & $-1.149 \times 10^{12}$ & $-3.681 \times 10^{12}$ & $-4.795 \times 10^{12}$ & $-9.177 \times 10^{12}$ & $2.268 \times 10^{12}$ \\
\hline 8 & $-3.665 \times 10^{12}$ & $-7.589 \times 10^{12}$ & $-7.751 \times 10^{12}$ & $-1.508 \times 10^{12}$ & $-4.290 \times 10^{12}$ \\
\hline 9 & $-1.148 \times 10^{12}$ & $-1.537 \times 10^{12}$ & $-1.216 \times 10^{12}$ & $-1.969 \times 10^{12}$ & $-4.728 \times 10^{12}$ \\
\hline 10 & $-3.550 \times 10^{12}$ & $-3.069 \times 10^{12}$ & $-1.881 \times 10^{12}$ & $-2.540 \times 10^{12}$ & $-5.194 \times 10^{12}$ \\
\hline 11 & $-1.085 \times 10^{12}$ & $-6.062 \times 10^{12}$ & $-2.878 \times 10^{12}$ & $-3.247 \times 10^{12}$ & $-5.798 \times 10^{12}$ \\
\hline 12 & $-3.288 \times 10^{12}$ & $-1.186 \times 10^{12}$ & $-4.364 \times 10^{12}$ & $-4.112 \times 10^{12}$ & $-6.417 \times 10^{12}$ \\
\hline 13 & $-9.888 \times 10^{12}$ & $-2.304 \times 10^{12}$ & $-6.567 \times 10^{12}$ & $-5.167 \times 10^{12}$ & $-7.015 \times 10^{12}$ \\
\hline 14 & $-2.955 \times 10^{12}$ & $-4.445 \times 10^{12}$ & $-9.815 \times 10^{12}$ & $-6.448 \times 10^{12}$ & $-7.622 \times 10^{12}$ \\
\hline 15 & $-8.781 \times 10^{12}$ & $-7.276 \times 10^{12}$ & $4.898 \times 10^{12}$ & $6.787 \times 10^{12}$ & $5.366 \times 10^{12}$ \\
\hline 16 & $-2.597 \times 10^{12}$ & $-1.629 \times 10^{12}$ & $-2.158 \times 10^{12}$ & $-9.880 \times 10^{12}$ & $-8.844 \times 10^{12}$ \\
\hline 17 & $-7.650 \times 10^{12}$ & $-3.097 \times 10^{12}$ & $-3.177 \times 10^{12}$ & $-1.215 \times 10^{12}$ & $-9.460 \times 10^{12}$ \\
\hline 18 & $-2.245 \times 10^{12}$ & $-5.867 \times 10^{12}$ & $-4.662 \times 10^{12}$ & $-1.487 \times 10^{12}$ & $-1.008 \times 10^{12}$ \\
\hline 19 & $-6.568 \times 10^{12}$ & $-1.108 \times 10^{12}$ & $-6.816 \times 10^{12}$ & $-1.815 \times 10^{12}$ & $-1.070 \times 10^{12}$ \\
\hline 20 & $-1.916 \times 10^{12}$ & $-2.085 \times 10^{12}$ & $-9.935 \times 10^{12}$ & $-2.207 \times 10^{12}$ & $-1.132 \times 10^{12}$ \\
\hline 21 & $-5.573 \times 10^{12}$ & $-3.915 \times 10^{12}$ & $-1.444 \times 10^{12}$ & $-2.678 \times 10^{12}$ & $-1.194 \times 10^{12}$ \\
\hline 22 & $-1.617 \times 10^{12}$ & $-7.334 \times 10^{12}$ & $-2.094 \times 10^{12}$ & $-3.240 \times 10^{12}$ & $-1.256 \times 10^{12}$ \\
\hline 23 & $-4.684 \times 10^{12}$ & $-1.371 \times 10^{12}$ & $-3.030 \times 10^{12}$ & $-3.911 \times 10^{12}$ & $-1.318 \times 10^{12}$ \\
\hline 24 & $-1.354 \times 10^{12}$ & $-2.556 \times 10^{12}$ & $-4.376 \times 10^{12}$ & $-4.712 \times 10^{12}$ & $-1.381 \times 10^{12}$ \\
\hline 25 & $-3.905 \times 10^{12}$ & $-4.759 \times 10^{12}$ & $-6.307 \times 10^{12}$ & $-5.666 \times 10^{12}$ & $-1.443 \times 10^{12}$ \\
\hline 26 & $-1.125 \times 10^{12}$ & $-8.845 \times 10^{12}$ & $-9.074 \times 10^{12}$ & $-6.800 \times 10^{12}$ & $-1.506 \times 10^{12}$ \\
\hline 27 & $-3.235 \times 10^{12}$ & $-1.641 \times 10^{12}$ & $-1.304 \times 10^{12}$ & $-8.149 \times 10^{12}$ & $-1.568 \times 10^{12}$ \\
\hline 28 & $-9.290 \times 10^{12}$ & $-3.041 \times 10^{12}$ & $-1.870 \times 10^{12}$ & $-9.750 \times 10^{12}$ & $-1.631 \times 10^{12}$ \\
\hline 29 & $-2.664 \times 10^{12}$ & $-5.628 \times 10^{12}$ & $-2.678 \times 10^{12}$ & $-1.165 \times 10^{12}$ & $-1.694 \times 10^{12}$ \\
\hline 30 & $-7.631 \times 10^{12}$ & $-1.040 \times 10^{12}$ & $-3.832 \times 10^{12}$ & $-1.390 \times 10^{12}$ & $-1.756 \times 10^{12}$ \\
\hline
\end{tabular}

where $n$ denotes the finite Fourier cosine transform parameter and $W\left(r_{\mathrm{D}}, n, s\right)$ represents the finite Fourier cosine transform of $G\left(r_{\mathrm{D}}, \theta, s\right)$, as defined by

$W\left(r_{\mathrm{D}}, n, s\right)=\int_{0}^{\pi} G\left(r_{\mathrm{D}}, \theta, s\right) \cos (n \theta) \mathrm{d} \theta$

Such a transform is advantageous in that the inversion is directly given by the following formula [16]:

$G\left(r_{\mathrm{D}}, \theta, s\right)=\frac{1}{\pi} W\left(r_{\mathrm{D}}, 0, s\right)+\frac{2}{\pi} \sum_{n=1}^{\infty} W\left(r_{\mathrm{D}}, n, s\right) \cos (n \theta)$

The transformed ordinary differential equation (A.9) can be directly solved using the power series method. The governing equation (A.9), however, involves an advection term that generally leads to a numerical error in numerical calculation for large Péclet numbers. Chen et al. [4] suggested that the power series technique, with a change of variables to eliminate the first spatial derivative, is capable of solving the radial dispersion problem over a wide range of Péclet numbers. Therefore, a change of variables is used to eliminate the first spatial derivative and convert Eq. (A.9) to
$\frac{1}{P e} \frac{\mathrm{d}^{2} Z}{\mathrm{~d} r_{\mathrm{D}}^{2}}-\left(\frac{P e}{4}+\frac{1}{P e} \frac{X n^{2}}{r_{\mathrm{D}}^{2}}+\frac{2 r_{\mathrm{D}} R}{1-r_{\mathrm{WD}}^{2}}\right) Z=0$

where

$Z=\exp \left[\frac{P e}{2}\left(r_{\mathrm{D}}-1\right)\right] W$

The boundary conditions (A.10) and (A.11) are in terms of $Z$,

$\frac{1}{P e} \frac{\mathrm{d} Z}{\mathrm{~d} r_{\mathrm{D}}}-\left(\frac{1}{2}+\mu_{\mathrm{W}} s\right) Z=0$

and

$\frac{1}{P e} \frac{\mathrm{d} W\left(r_{\mathrm{D}}, n, s\right)}{\mathrm{d} r_{\mathrm{D}}}+W\left(r_{\mathrm{D}}, n, s\right)=F(n)$,

respectively.

The governing equation (A.14) is amenable to solution using the power series method [8]. While using the power series method to solve the transformed differential equation (A.14), one must consider that whether the variable-dependent coefficients of governing equation 
Table 7

The values of $\partial Z_{2}\left(r_{\mathrm{D}}, n, s\right) / \partial r_{\mathrm{D}}$ for various $r_{\mathrm{D}}$ and $n$ at $s=3(P e=10)$

\begin{tabular}{|c|c|c|c|c|c|}
\hline$n$ & $r_{\mathrm{D}}=0.2$ & $r_{\mathrm{D}}=0.4$ & $r_{\mathrm{D}}=0.6$ & $r_{\mathrm{D}}=0.8$ & $r_{\mathrm{D}}=1.0$ \\
\hline 0 & $1.732 \times 10^{12}$ & $6.280 \times 10^{12}$ & $2.925 \times 10^{12}$ & $1.579 \times 10^{12}$ & $9.733 \times 10^{12}$ \\
\hline 1 & $2.421 \times 10^{12}$ & $1.071 \times 10^{12}$ & $5.074 \times 10^{12}$ & $2.758 \times 10^{12}$ & $1.707 \times 10^{12}$ \\
\hline 2 & $-1.469 \times 10^{12}$ & $2.090 \times 10^{12}$ & $1.201 \times 10^{12}$ & $6.695 \times 10^{12}$ & $4.198 \times 10^{12}$ \\
\hline 3 & $-2.491 \times 10^{12}$ & $-9.155 \times 10^{12}$ & $-4.691 \times 10^{12}$ & $-2.688 \times 10^{12}$ & $-1.722 \times 10^{12}$ \\
\hline 4 & $-1.993 \times 10^{12}$ & $6.235 \times 10^{12}$ & $3.743 \times 10^{12}$ & $2.260 \times 10^{12}$ & $1.492 \times 10^{12}$ \\
\hline 5 & $-9.838 \times 10^{12}$ & $1.695 \times 10^{12}$ & $1.521 \times 10^{12}$ & $9.856 \times 10^{12}$ & $6.771 \times 10^{12}$ \\
\hline 6 & $-3.370 \times 10^{12}$ & $-7.290 \times 10^{12}$ & $4.882 \times 10^{12}$ & $3.516 \times 10^{12}$ & $2.536 \times 10^{12}$ \\
\hline 7 & $-1.101 \times 10^{12}$ & $-4.133 \times 10^{12}$ & $-1.113 \times 10^{12}$ & $-8.347 \times 10^{12}$ & $-6.363 \times 10^{12}$ \\
\hline 8 & $-3.514 \times 10^{12}$ & $-5.203 \times 10^{12}$ & $1.026 \times 10^{12}$ & $9.125 \times 10^{12}$ & $7.423 \times 10^{12}$ \\
\hline 9 & $-1.103 \times 10^{12}$ & $-1.234 \times 10^{12}$ & $3.316 \times 10^{12}$ & $3.856 \times 10^{12}$ & $3.373 \times 10^{12}$ \\
\hline 10 & $-3.418 \times 10^{12}$ & $-2.541 \times 10^{12}$ & $1.070 \times 10^{12}$ & $2.399 \times 10^{12}$ & $2.273 \times 10^{12}$ \\
\hline 11 & $-1.047 \times 10^{12}$ & $-5.085 \times 10^{12}$ & $1.115 \times 10^{12}$ & $3.610 \times 10^{12}$ & $3.719 \times 10^{12}$ \\
\hline 12 & $-3.179 \times 10^{12}$ & $-1.008 \times 10^{12}$ & $-2.757 \times 10^{12}$ & $9.687 \times 10^{12}$ & $1.279 \times 10^{12}$ \\
\hline 13 & $-9.578 \times 10^{12}$ & $-1.975 \times 10^{12}$ & $-4.248 \times 10^{12}$ & $1.849 \times 10^{12}$ & $2.558 \times 10^{12}$ \\
\hline 14 & $-2.867 \times 10^{12}$ & $-3.842 \times 10^{12}$ & $-6.584 \times 10^{12}$ & $1.629 \times 10^{12}$ & $2.617 \times 10^{12}$ \\
\hline 15 & $-1.066 \times 10^{12}$ & $-6.291 \times 10^{12}$ & $-3.292 \times 10^{12}$ & $-7.218 \times 10^{12}$ & $-1.087 \times 10^{12}$ \\
\hline 16 & $-2.528 \times 10^{12}$ & $-1.428 \times 10^{12}$ & $-1.527 \times 10^{12}$ & $-7.451 \times 10^{12}$ & $-4.285 \times 10^{12}$ \\
\hline 17 & $-7.455 \times 10^{12}$ & $-2.732 \times 10^{12}$ & $-2.285 \times 10^{12}$ & $-6.470 \times 10^{12}$ & $-4.746 \times 10^{12}$ \\
\hline 18 & $-2.190 \times 10^{12}$ & $-5.206 \times 10^{12}$ & $-3.402 \times 10^{12}$ & $-7.658 \times 10^{12}$ & $3.915 \times 10^{12}$ \\
\hline 19 & $-6.415 \times 10^{12}$ & $-9.881 \times 10^{12}$ & $-5.043 \times 10^{12}$ & $-1.048 \times 10^{12}$ & $-1.488 \times 10^{12}$ \\
\hline 20 & $-1.873 \times 10^{12}$ & $-1.869 \times 10^{12}$ & $-7.444 \times 10^{12}$ & $-1.235 \times 10^{12}$ & $-1.853 \times 10^{12}$ \\
\hline 21 & $-5.454 \times 10^{12}$ & $-3.525 \times 10^{12}$ & $-1.095 \times 10^{12}$ & $-1.529 \times 10^{12}$ & $-8.763 \times 10^{12}$ \\
\hline 22 & $-1.584 \times 10^{12}$ & $-6.630 \times 10^{12}$ & $-1.605 \times 10^{12}$ & $-1.889 \times 10^{12}$ & $-6.922 \times 10^{12}$ \\
\hline 23 & $-4.591 \times 10^{12}$ & $-1.244 \times 10^{12}$ & $-2.346 \times 10^{12}$ & $-2.325 \times 10^{12}$ & $-5.172 \times 10^{12}$ \\
\hline 24 & $-1.328 \times 10^{12}$ & $-2.328 \times 10^{12}$ & $-3.418 \times 10^{12}$ & $-2.854 \times 10^{12}$ & $-5.831 \times 10^{12}$ \\
\hline 25 & $-3.834 \times 10^{12}$ & $-4.349 \times 10^{12}$ & $-4.970 \times 10^{12}$ & $-3.491 \times 10^{12}$ & $-6.242 \times 10^{12}$ \\
\hline 26 & $-1.105 \times 10^{12}$ & $-8.107 \times 10^{12}$ & $-7.209 \times 10^{12}$ & $-4.259 \times 10^{12}$ & $-6.697 \times 10^{12}$ \\
\hline 27 & $-3.179 \times 10^{12}$ & $-1.509 \times 10^{12}$ & $-1.043 \times 10^{12}$ & $-5.181 \times 10^{12}$ & $-7.090 \times 10^{12}$ \\
\hline 28 & $-9.135 \times 10^{12}$ & $-2.803 \times 10^{12}$ & $-1.507 \times 10^{12}$ & $-6.288 \times 10^{12}$ & $-7.565 \times 10^{12}$ \\
\hline 29 & $-2.621 \times 10^{12}$ & $-5.201 \times 10^{12}$ & $-2.174 \times 10^{12}$ & $-7.615 \times 10^{12}$ & $-8.040 \times 10^{12}$ \\
\hline 30 & $-7.512 \times 10^{12}$ & $-9.636 \times 10^{12}$ & $-3.129 \times 10^{12}$ & $-9.202 \times 10^{12}$ & $-8.521 \times 10^{12}$ \\
\hline
\end{tabular}

are analytical or not. There are two different type power series solutions for the governing equation (A.14): Case 1 , the coefficient is analytical for $n=0$; and Case 2, the coefficient is not analytical for $n>0$. Thus, the solution is obtained by direct application of the power series method for Case 1. The other solution is yielded using the extended power series method, or the Frobenius method, for Case 2 [10,21]. The procedures for the two approaches are described as follows:

Case 1: $n=0$, the coefficient is analytical

Rewriting the governing equation (A.14) for $n=0$ yields

$\frac{1}{P e} \frac{\mathrm{d}^{2} Z}{\mathrm{~d} r_{\mathrm{D}}^{2}}-\left(\frac{P e}{4}+\frac{2 r_{\mathrm{D}} R}{1-r_{\mathrm{WD}}^{2}}\right) Z=0$

First, the solution of Eq. (A.17) is assumed in the form of a power series with unknown coefficients,

$Z\left(r_{\mathrm{D}}, 0, s\right)=\sum_{m=0}^{\infty} a_{m} r_{\mathrm{D}}^{m}$

Termwise differentiation gives

$$
\begin{aligned}
& \frac{\mathrm{d} Z\left(r_{\mathrm{D}}, 0, s\right)}{\mathrm{d} r_{\mathrm{D}}}=\sum_{1}^{\infty} m a_{m} r_{\mathrm{D}}^{m-1} \\
& \frac{\mathrm{d} Z\left(r_{\mathrm{D}}, 0, s\right)}{\mathrm{d} r_{\mathrm{D}}^{2}}=\sum_{2}^{\infty} m(m-1) a_{m} r_{\mathrm{D}}^{m-2}
\end{aligned}
$$

These expressions are substituted into Eq. (A.17), yielding

$\frac{1}{P e} \sum_{2}^{\infty} m(m-1) a_{m} r_{\mathrm{D}}^{m-2}-\left(\frac{P e}{4}+\frac{2 R s}{1-r_{\mathrm{WD}}^{2}} r_{\mathrm{D}}\right) \sum_{0}^{\infty} a_{m} r_{\mathrm{D}}^{m}=0$

By shifting summation indices, we obtain

$$
\begin{gathered}
\frac{1}{P e} \sum_{0}^{\infty}(m+2)(m+1) a_{m+2} r_{\mathrm{D}}^{m}-\frac{P e}{4} \sum_{0}^{\infty} a_{m} r_{\mathrm{D}}^{m} \\
-\frac{2 R s}{1-r_{\mathrm{WD}}^{2}} \sum_{1}^{\infty} a_{m-1} r_{\mathrm{D}}^{m}=0
\end{gathered}
$$

The coefficient of each power of $r_{\mathrm{D}}$ to set to zero. For $m=0$ this yields 
Table 8

The values of $\partial Z_{2}\left(r_{\mathrm{D}}, n, s\right) / \partial r_{\mathrm{D}}$ for various $r_{\mathrm{D}}$ and $n$ at $s=3(P e=60)$

\begin{tabular}{|c|c|c|c|c|c|}
\hline$n$ & $r_{\mathrm{D}}=0.2$ & $r_{\mathrm{D}}=0.4$ & $r_{\mathrm{D}}=0.6$ & $r_{\mathrm{D}}=0.8$ & $r_{\mathrm{D}}=1.0$ \\
\hline 0 & $2.308 \times 10^{12}$ & $1.345 \times 10^{12}$ & $9.778 \times 10^{12}$ & $8.812 \times 10^{12}$ & $9.779 \times 10^{12}$ \\
\hline 1 & $4.123 \times 10^{12}$ & $2.435 \times 10^{12}$ & $1.779 \times 10^{12}$ & $1.607 \times 10^{12}$ & $1.786 \times 10^{12}$ \\
\hline 2 & $-1.326 \times 10^{12}$ & $-8.147 \times 10^{12}$ & $-6.037 \times 10^{12}$ & $-5.491 \times 10^{12}$ & $-6.127 \times 10^{12}$ \\
\hline 3 & $-4.154 \times 10^{12}$ & $-2.728 \times 10^{12}$ & $-2.069 \times 10^{12}$ & $-1.904 \times 10^{12}$ & $-2.139 \times 10^{12}$ \\
\hline 4 & $1.915 \times 10^{12}$ & $1.380 \times 10^{12}$ & $1.081 \times 10^{12}$ & $1.012 \times 10^{12}$ & $1.148 \times 10^{12}$ \\
\hline 5 & $-5.434 \times 10^{12}$ & $-4.409 \times 10^{12}$ & $-3.602 \times 10^{12}$ & $-3.441 \times 10^{12}$ & $-3.951 \times 10^{12}$ \\
\hline 6 & $-1.438 \times 10^{12}$ & $-1.347 \times 10^{12}$ & $-1.157 \times 10^{12}$ & $-1.134 \times 10^{12}$ & $-1.322 \times 10^{12}$ \\
\hline 7 & $-5.281 \times 10^{12}$ & $-5.842 \times 10^{12}$ & $-5.330 \times 10^{12}$ & $-5.380 \times 10^{12}$ & $-6.385 \times 10^{12}$ \\
\hline 8 & $-5.551 \times 10^{12}$ & $-7.421 \times 10^{12}$ & $-7.248 \times 10^{12}$ & $-7.573 \times 10^{12}$ & $-9.172 \times 10^{12}$ \\
\hline 9 & $-3.531 \times 10^{12}$ & $-5.817 \times 10^{12}$ & $-6.134 \times 10^{12}$ & $-6.662 \times 10^{12}$ & $-8.257 \times 10^{12}$ \\
\hline 10 & $3.854 \times 10^{12}$ & $8.217 \times 10^{12}$ & $9.432 \times 10^{12}$ & $1.070 \times 10^{12}$ & $1.360 \times 10^{12}$ \\
\hline 11 & $2.577 \times 10^{12}$ & $6.835 \times 10^{12}$ & $8.607 \times 10^{12}$ & $1.023 \times 10^{12}$ & $1.339 \times 10^{12}$ \\
\hline 12 & $-5.255 \times 10^{12}$ & $-1.766 \times 10^{12}$ & $-2.459 \times 10^{12}$ & $-3.078 \times 10^{12}$ & $-4.154 \times 10^{12}$ \\
\hline 13 & $2.224 \times 10^{12}$ & $1.844 \times 10^{12}$ & $2.859 \times 10^{12}$ & $3.785 \times 10^{12}$ & $5.281 \times 10^{12}$ \\
\hline 14 & $1.772 \times 10^{12}$ & $1.805 \times 10^{12}$ & $3.139 \times 10^{12}$ & $4.410 \times 10^{12}$ & $6.379 \times 10^{12}$ \\
\hline 15 & $-1.965 \times 10^{12}$ & $-1.605 \times 10^{12}$ & $-3.152 \times 10^{12}$ & $-4.720 \times 10^{12}$ & $-7.094 \times 10^{12}$ \\
\hline 16 & $-1.151 \times 10^{12}$ & $1.628 \times 10^{12}$ & $3.633 \times 10^{12}$ & $5.821 \times 10^{12}$ & $9.114 \times 10^{12}$ \\
\hline 17 & $-3.516 \times 10^{12}$ & $8.386 \times 10^{12}$ & $2.140 \times 10^{12}$ & $3.682 \times 10^{12}$ & $6.020 \times 10^{12}$ \\
\hline 18 & $-1.081 \times 10^{12}$ & $9.649 \times 10^{12}$ & $2.833 \times 10^{12}$ & $5.252 \times 10^{12}$ & $8.989 \times 10^{12}$ \\
\hline 19 & $-3.280 \times 10^{12}$ & $3.902 \times 10^{12}$ & $1.325 \times 10^{12}$ & $2.657 \times 10^{12}$ & $4.771 \times 10^{12}$ \\
\hline 20 & $-9.854 \times 10^{12}$ & $3.476 \times 10^{12}$ & $1.372 \times 10^{12}$ & $2.987 \times 10^{12}$ & $5.640 \times 10^{12}$ \\
\hline 21 & $-2.946 \times 10^{12}$ & $2.081 \times 10^{12}$ & $9.602 \times 10^{12}$ & $2.275 \times 10^{12}$ & $4.528 \times 10^{12}$ \\
\hline 22 & $-8.769 \times 10^{12}$ & $4.244 \times 10^{12}$ & $2.300 \times 10^{12}$ & $5.953 \times 10^{12}$ & $1.251 \times 10^{12}$ \\
\hline 23 & $-2.599 \times 10^{12}$ & $1.747 \times 10^{12}$ & $1.116 \times 10^{12}$ & $3.167 \times 10^{12}$ & $7.047 \times 10^{12}$ \\
\hline 24 & $-7.677 \times 10^{12}$ & $4.701 \times 10^{12}$ & $3.560 \times 10^{12}$ & $1.110 \times 10^{12}$ & $2.620 \times 10^{12}$ \\
\hline 25 & $-2.260 \times 10^{12}$ & $9.379 \times 10^{12}$ & $8.490 \times 10^{12}$ & $2.918 \times 10^{12}$ & $7.320 \times 10^{12}$ \\
\hline 26 & $-6.633 \times 10^{12}$ & $-2.294 \times 10^{12}$ & $-2.454 \times 10^{12}$ & $-9.324 \times 10^{12}$ & $-2.491 \times 10^{12}$ \\
\hline 27 & $-1.941 \times 10^{12}$ & $1.181 \times 10^{12}$ & $1.547 \times 10^{12}$ & $6.514 \times 10^{12}$ & $1.857 \times 10^{12}$ \\
\hline 28 & $-5.668 \times 10^{12}$ & $1.345 \times 10^{12}$ & $2.718 \times 10^{12}$ & $1.272 \times 10^{12}$ & $3.879 \times 10^{12}$ \\
\hline 29 & $-1.651 \times 10^{12}$ & $-1.270 \times 10^{12}$ & $-8.639 \times 10^{12}$ & $-4.505 \times 10^{12}$ & $-1.471 \times 10^{12}$ \\
\hline 30 & $-4.798 \times 10^{12}$ & $-5.915 \times 10^{12}$ & $-9.794 \times 10^{12}$ & $-5.705 \times 10^{12}$ & $-2.000 \times 10^{12}$ \\
\hline
\end{tabular}

$a_{2}=\frac{P e^{2}}{8} a_{0}$

and in general and when $m=1,2,3, \ldots$

$a_{m+2}=\frac{P e}{(m+2)(m+1)}\left(\frac{P e}{4} a_{m}+\frac{2 R s}{1-r_{\mathrm{WD}}^{2}} a_{m-1}\right)$

By inserting the value for the coefficients into (A.18), the general solution is obtained

$Z\left(r_{\mathrm{D}}, 0, s\right)=b_{1} Z_{1}\left(r_{\mathrm{D}}, 0, s\right)+b_{2} Z_{2}\left(r_{\mathrm{D}}, 0, s\right)$

where $Z_{1}\left(r_{\mathrm{D}}, 0, s\right)$ and $Z_{2}\left(r_{\mathrm{D}}, 0, s\right)$ are two linearly independent functions which are of the form of (A.18), with coefficients determined by (A.23) and (A.24) and set $a_{0}=1, a_{1}=0$ or $a_{0}=0, a_{1}=1$, respectively.

Case 2: $n>0$, the coefficient is not analytical

The governing equation (A.14), except for $n=0$, subjected to boundary conditions (A.15) and (A.16) is solved using the Frobenius power series method. First, assume the solution of equation (A.14) has the form of power series with undetermined coefficients,

$Z=\sum_{m=0}^{\infty} a_{m} r_{\mathrm{D}}^{m+r}$ and insert this series and the series obtained by termwise differentiation,

$\frac{\mathrm{d} Z}{\mathrm{~d} r_{\mathrm{D}}}=\sum_{m=0}^{\infty}(m+r) a_{m} r_{\mathrm{D}}^{m+r-1}$

$\frac{\mathrm{d}^{2} Z}{\mathrm{~d} r_{\mathrm{D}}}=\sum_{m=0}^{\infty}(m+r)(m+r-1) a_{m} r_{\mathrm{D}}^{m+r-2}$

into Eq. (A.14). This yields:

$$
\begin{aligned}
P e & \cdot r_{\mathrm{D}}^{2} \sum_{m=0}^{\infty}(m+r)(m+r-1) a_{m} r_{\mathrm{D}}^{m+r-2} \\
& -\left(\frac{X n^{2}}{P e}+\frac{P e}{4} r_{\mathrm{D}}^{2}+\frac{2 R s}{1-r_{\mathrm{WD}}^{2}} r_{\mathrm{D}}^{3}\right) \sum_{m=0}^{\infty} a_{m} r_{\mathrm{D}}^{m+r}=0
\end{aligned}
$$

By shifting summation indices, we obtain

$$
\begin{aligned}
P e & \sum_{m=0}^{\infty}(m+r)(m+r-1) a_{m} r_{\mathrm{D}}^{m+r}-\frac{X n^{2}}{P e} \sum_{m=0}^{\infty} a_{m} r_{\mathrm{D}}^{m+r} \\
& -\frac{P e}{4} \sum_{m=2}^{\infty} a_{m-2} r_{\mathrm{D}}^{m+r}-\frac{2 R s}{1-r_{\mathrm{WD}}^{2}} \sum_{m=3}^{\infty} a_{m-3} r_{\mathrm{D}}^{m+r}=0
\end{aligned}
$$


Equating the coefficient of each power of $r_{\mathrm{D}}$ to zero yields a general formula for all $m$.

$$
\begin{aligned}
& \frac{1}{P e} r(r-1) a_{0}-\frac{X n^{2}}{P e} a_{0}=0 \quad(m=0) \\
& \frac{1}{P e}(r+1) r a_{1}-\frac{X n^{2}}{P e} a_{1}=0 \quad(m=1) \\
& \frac{1}{P e}(r+2)(r+1) a_{2}-\frac{X n^{2}}{P e} a_{2}-\frac{P e}{4} a_{0}=0 \quad(m=2) \\
& \frac{1}{P e}(r+m)(r+m-1) a_{m}-\frac{X n^{2}}{P e} a_{m}-\frac{P e}{4} a_{m-2} \\
& -\frac{2 R s}{1-r_{\mathrm{WD}}^{2}} a_{m-3}=0 \quad(m=3,4,5, \ldots)
\end{aligned}
$$

From (A.31), the indicial equation is obtained

$r(r-1)-X n^{2}=0$

The roots are $r_{1}=\frac{1+\sqrt{1+4 X n^{2}}}{2}$ and $r_{2}=\frac{1-\sqrt{1+4 X n^{2}}}{2}$, respectively.

Eqs. (A.32)-(A.34) yield

$$
a_{1}=0
$$

$a_{2}=\frac{P e^{2}}{4\left[(r+2)(r+1)-X n^{2}\right]} a_{0}=0$

$a_{m}=\frac{\frac{P e^{2}}{4} a_{m-2}+P e a_{m-3}}{(r+m)(r+m-1)-X n^{2}} \quad(m=3,4,5, \ldots)$

By inserting the value for the coefficients into (A.26) the general solution is obtained as

$Z\left(r_{\mathrm{D}}, n, s\right)=b_{1} Z_{1}\left(r_{\mathrm{D}}, n, s\right)+b_{2} Z_{2}\left(r_{\mathrm{D}}, n, s\right)$

where $Z_{1}\left(r_{\mathrm{D}}, n, s\right)$ and $Z_{2}\left(r_{\mathrm{D}}, n, s\right)$ are two linearly independent functions that have the form given in (A.26), with coefficients determined by (A.36)-(A.38) and set $r=r_{1}$ or $r=r_{2}$, respectively.

Particular solutions can be obtained by straightforward application of boundary conditions (A.15) and (A.16) to the general solution given by (A.25) and (A.39). Thus, the Laplace-finite Fourier cosine transform power series solution may be written as

$$
\begin{aligned}
& W\left(r_{\mathrm{D}}, n, s\right)=\exp \left[\frac{P e}{2}\left(1-r_{\mathrm{D}}\right)\right] \\
& \times \frac{-U\left(S P_{21}-A P_{22}\right) Z_{1}\left(r_{\mathrm{D}}, n, s\right)+U\left(S P_{11}-A P_{12}\right) Z_{2}\left(r_{\mathrm{D}}, n, s\right)}{\left(S P_{11}-A P_{12}\right)\left(T Q_{21}+A Q_{22}\right)-\left(S P_{21}-A P_{22}\right)\left(T Q_{11}+A Q_{12}\right)}
\end{aligned}
$$

where

$$
S=\frac{1}{2}+\mu_{\mathrm{W}} s
$$

$$
\begin{aligned}
& T=\frac{1}{2} \\
& U=F(n) \\
& P_{11}=Z_{1}\left(r_{\mathrm{WD}}, n, s\right) \\
& P_{21}=Z_{2}\left(r_{\mathrm{WD}}, n, s\right) \\
& P_{12}=\frac{\partial Z_{1}\left(r_{\mathrm{WD}}, n, s\right)}{\partial r_{\mathrm{D}}} \\
& P_{22}=\frac{\partial Z_{2}\left(r_{\mathrm{WD}}, n, s\right)}{\partial r_{\mathrm{D}}} \\
& Q_{11}=Z_{1}(1, n, s) \\
& Q_{21}=Z_{2}(1, n, s) \\
& Q_{12}=\frac{\partial Z_{1}(1, n, s)}{\partial r_{\mathrm{D}}} \\
& Q_{22}=\frac{\partial Z_{2}(1, n, s)}{\partial r_{\mathrm{D}}}
\end{aligned}
$$

Solutions in the original domain $C\left(r_{\mathrm{D}}, \theta, t_{\mathrm{D}}\right)$ are the Laplace and finite Fourier cosine inversion of $W\left(r_{\mathrm{D}}, n, s\right)$. The finite Fourier cosine inverse transform is performed first. In addition, the Laplace inverse of (A.40) has to be determined numerically. A FORTRAN subroutine, DINLAP/INLAP, provided by IMSL Subroutine Library [18] and based on the de Hoog et al. [6] algorithm, is employed to perform the numerical Laplace inversion.

\section{Appendix B}

Tables 3-8 present the computed values of the function, $Z_{2}\left(r_{\mathrm{D}}, n, s\right)$, and its first derivatives with respect to $r_{\mathrm{D}}, \partial Z_{2}\left(r_{\mathrm{D}}, n, s\right) / \partial r_{\mathrm{D}}$ for various $n$ and $r_{\mathrm{D}}$ values when $s$ is fixed at 3 .

\section{References}

[1] Becker MW, Charbeneau RJ. First-passage-time transfer functions for groundwater tracer tests conducted in radially convergent flow. J Contam Hydrol 2000;40:299-310.

[2] Chen JS, Liu CW, Chen CS, Yeh HD. A Laplace transform solution for tracer test in a radially convergent flow with upstream dispersion. J Hydrol 1996;183:263-75.

[3] Chen JS, Chen CS, Gau HS, Liu CW. A two-well method to evaluate transverse dispersivity for tracer tests in a radially convergent flow field. J Hydrol 1999;223:175-97.

[4] Chen JS, Liu CW, Liao CM. A novel analytical power series solution for solute transport in a radially convergent flow field. J Hydrol 2002;266:120-38.

[5] Chen JS, Liu CW, Chen CS, Liao CM. Effect of well bore mixing volume on nonaxisymmetrical transport in a convergent radial tracer test. J Hydrol 2003;277:61-73. 
[6] de Hoog FR, Knight JH, Stokes AN. An improved method of Laplace transforms using a Fourier series approximation. SIAM J Sci Stat Comput 1982;3(3):357-66.

[7] Drost W, Klotz D, Koch A, Moser H, Neumaier F, Rauert W. Point dilution methods of investigating ground water flow by means of radioisotopes. Water Resour Res 1968;4(1):125-46.

[8] Gustafan KE. Introduction to partial differential equations and Hilbert space methods. New York: John Wiley and Sons; 1980.

[9] Hodgkinson DP, Lever DA. Interpretation of a field experiment on transport of sorbed and non-sorbed tracers through a fracture in crystalline rock. Radioact Waste Manage Nucl Fuel Cycle 1983;4(2):129-58.

[10] Kreyszig E. Advanced engineering mathematics. New York: John Wiley and Sons; 1968.

[11] Mackay DM, Bianchi-Mosquera G, Kopania AW, Kianjah H. A forced-gradient experiment on solute transport in the Borden aquifer. 1. Experimental methods and moment analysis of results. Water Resour Res 1994;30(2):369-83.

[12] Moench AF. Convergent radial dispersion: a Laplace transform solution for aquifer tracer testing. Water Resour Res 1989; 25(3):439-47.

[13] Moench AF. Convergent radial dispersion: a note on evaluation of the Laplace transform solution. Water Resour Res 1991;27(12): 3261-4.
[14] Moench AF. Convergent radial dispersion in a double-porosity aquifer with fracture skin: analytical solution and application to a field experiment in fractured chalk. Water Resour Res 1995; 31(8):1823-35.

[15] Sauty JP. An analysis of hydrodispersive transfer in aquifers. Water Resour Res 1980;16(1):145-58.

[16] Sneddon IN. The use of integral transform. New York: McGrawHill; 1972.

[17] Thorbjarnson K, Mackay DM. A forced gradient experiment on solute transport in the Borden aquifer. 2. Transport and dispersion of the conservative tracer. Water Resour Res 1994;30(2):38599.

[18] Visual Numerical, Inc. IMSL User's Manual. Houston, TX, vol. 1, 1994, p. 159-61.

[19] Wang HQ, Crampon N. Method for interpreting tracer experiments in radial flow using modified analytical solutions. J Hydrol 1995;165:11-31.

[20] Welty C, Gelhar LW. Evaluation of longitudinal dispersivity from nonuniform flow tracer tests. J Hydrol 1994;153:71-102.

[21] Wylie CR, Barrett LC. Advanced engineering mathematics. New York: McGraw-Hill Higher Education; 1995.

[22] Zlotnik VA, Logan JD. Boundary conditions for convergent radial tracer tests and the effect of well bore mixing volume. Water Resour Res 1996;32(7):2323-8. 